\title{
White-coat hypertension and albuminuria; lessons from the Hisayama and Ohasama studies
}

\author{
Yasuyuki Nagasawa ${ }^{1} \cdot$ Aritoshi Kida $^{1} \cdot$ Yukiko Hasuike $^{1} \cdot$ Takahiro Kuragano $^{1} \cdot$ Takeshi Nakanishi $^{1}$
}

Received: 29 September 2017 / Revised: 31 October 2017 / Accepted: 31 October 2017 / Published online: 18 April 2018

(c) The Japanese Society of Hypertension 2018

\section{Implications of white-coat hypertension}

Ambulatory blood pressure monitoring was used to create four categories of blood pressure, true normotension (NT), white-coat hypertension (WCHT), masked hypertension (MHT), and sustained hypertension (SHT), from one simple office blood pressure measurement [1]. The Ohasama study reported that a general population with WCHT and MHT had a higher incidence of mortality from cardiovascular disease (CVD) than the one with NT (Fig. 1a) [2]. Compared with the NT population, the WCHT population seemed to have a higher incidence of CVD mortality (hazards ratio 1.54 (0.73-3.21)), whereas the SHT population had a much higher incidence than the WCHT population. However, a meta-analysis of the data, which included the Ohasama study, failed to demonstrate the significance of WCHT [3]. This meta-analysis thus rendered the meaning of WCHT controversial. Recently, a new meta-analysis that analyzed 20,455 subjects across 23 cohort studies on an individual level demonstrated that WCHT was associated with a $38 \%$ increased risk of CVD and a $20 \%$ increase in total mortality [4]. Another meta-analysis also supported a significant risk of cardiovascular events associated with WCHT [5]. Moreover, in chronic kidney disease patients, WCHT and MHT were also reported to be risk factors for CVD [6]. At this point, the clinical evidence suggests that the risk of mortality and CVD events associated with WCHT lies between those with NT and SHT. The prevalence of WCHT was $32.7 \%$, as reported in the Spanish ambulatory blood pressure registry, whereas that of SHT was $44.5 \%$ [7].

Yasuyuki Nagasawa

nagasawa@hyo-med.ac.jp

1 Department of Internal Medicine, Division of Kidney and Dialysis, Hyogo College of Medicine, 1-1, Mukogawa-Cho,

Nishinomiya 663-8501, Japan
The implications of WCHT have been evaluated from several perspectives in relation to CVD and mortality [8]. The Hisayama study, one of the most famous cohort studies, reported that WCHT was associated with carotid atherosclerosis (Fig. 1b) [9]. In this report, the average thickness of intima-media in cases of WCHT was the same as that for SHT, which was significantly higher than that in NT. This finding was supported by a meta-analysis [10]. Retinal vascular abnormalities in WCHT were reported to be the same as those in SHT [11]. Development of atherosclerosis induced by WCHT should result in SHT. Indeed, WCHT led to the development of SHT at a higher rate than NT (odds ratio 2.51; $P<0.0001$ ) [12]. However, detailed clinical features remain unclear because the meaning of WCHT has only recently been established.

\section{Albuminuria in white-coat hypertension}

In this current issue, the Hisayama cohort study reported that WCHT was associated with a significantly increased age- and sex-adjusted prevalence of albuminuria, compared to NT ( 26.3 vs. $14.1 \%, P<0.001)$, although this research was from a cross-sectional study [13]. Interestingly, the effect of WCHT on the prevalence of albuminuria was similar to the effect of WCHT on CVD events and carotid atherosclerosis (Fig. 1a, b). This consistent effect of WCHT on several atherosclerotic diseases could make the pathogenicity of WCHT convincing.

Albuminuria was basically considered to be the result of endothelial dysfunction, which means that albuminuria was considered to be the result of hypertension. However, a slight increase in albuminuria within the normal range predicted hypertension in the future, suggesting that albuminuria might be the cause of hypertension [14]. At this point, it is difficult to distinguish which is the causal factor, hypertension, or albuminuria [15]. With respect to WCHT, there is no consistent evidence supporting endothelial dysfunction. Evaluation of endothelial function by brachial artery flow-mediated 


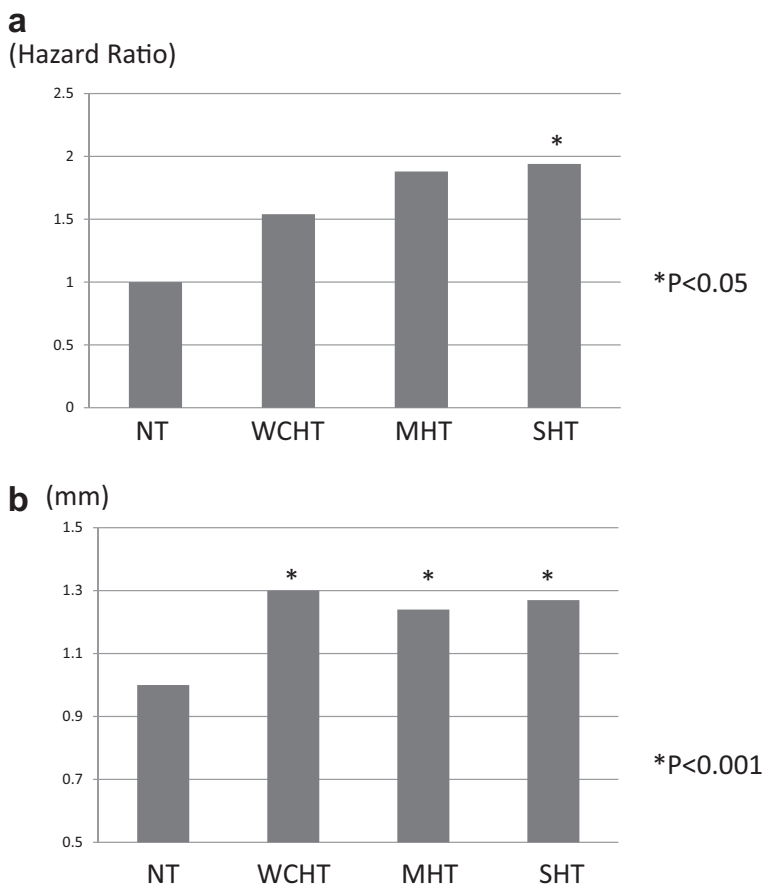

Fig. 1 a Hazard risk for morality related with cardiovascular diseases in the Ohasama study. The Ohasama cohort study reported that the hazard risk for cardiovascular diseases in those with WCHT appeared higher than that in NT $(1.54,95 \%$ CI $(0.73-3.72))$, whereas the hazard risk in those with MHT and SHT was 1.88 (0.95-3.72) and 1.94 (1.04-3.61), respectively. Figure adapted from Ohkubo et al [2]. b Maximum thickness of carotid atherosclerosis in the Hisayama study. The Hisayama study reported that the geometric average of intimamedia thickness in NT (1.15 mm (95\% CI, 1.13-1.17)), after adjustment for age, sex, diabetes mellitus, total cholesterol, high-density lipoprotein cholesterol, body mass index, smoking, drinking, exercise, and medications, was significantly less than that in WCHT $(1.30 \mathrm{~mm}$ (95\% CI 1.24-1.37), $P<0.001)$, MHT $(1.24 \mathrm{~mm} \quad(1.21-1.28)$, $P<0.001)$ and SHT $(1.27 \mathrm{~mm}(1.24-1.31), P<0.001)$. Figure adapted from Fukuhara et.al [9]. MHT masked hypertension, NT normotension, SHT sustained hypertension, WCHT white-coat hypertension

vasodilation has provided conflicting results $[16,17]$. More directly, Oliveras et al. [18] failed to demonstrate a relationship between albuminuria and WCHT. The effect on albuminuria associated with WCHT was similar to the effect on carotid atherosclerosis and CVD events, which may reflect the relationship between WCHT and albuminuria, and more importantly, the pathogenicity of WCHT.

\section{Mechanism by which white-coat hypertension is associated with albuminuria}

The clinical pathogenicity of WCHT itself was established recently [10]. Therefore, the mechanism by which WCHT causes an increase in CVD events, resulting in greater mortality, has ultimately remained unknown $[19,20]$. However, there are several mechanisms to consider that may explain why WCHT was associated with albuminuria (Fig. 2). WCHT
Sympathetic nerve activity

Metabolic syndrome

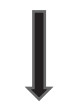

White-Coat Hypertension

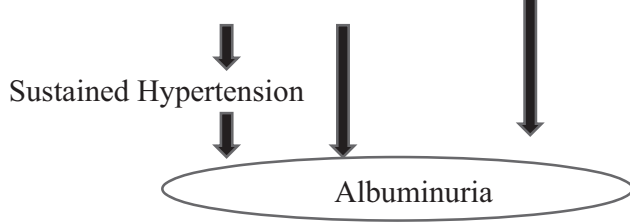

?

Progression of CKD?

Fig. 2 Potential mechanisms by which white-coat hypertension is associated with albuminuria. Hypersensitive sympathetic nerve activity and metabolic syndrome are reportedly associated with WCHT. At the same time, both of these could cause albuminuria. Those with WCHT have a high incidence of SHT, which might cause albuminuria. There is still some possibility that WCHT might directly induce albuminuria. It is unknown whether albuminuria might result in the progression of chronic kidney diseases

was defined as an increase in blood pressure at the doctor's office, which obviously suggested that patients with WCHT had hypersensitive sympathetic nervous activity. Indeed, there are many reports that support this hypersensitive activity in those with WCHT. Those with WCHT had significantly higher sympathetic nervous activity than those with NT, when measured by microneurography [21]. Those with WCHT had a higher ratio of high-frequency to low-frequency heart rate variability than those with NT $[22,23]$. More directly, the WCHT group had a greater blood pressure response to mental stress than the NT group [24]. In the first mechanism, this hypersensitive sympathetic activity was believed to be a strong risk factor for CKD, CVD, and mortality [25-28], just as this hypersensitive activity may cause WCHT. In the second mechanism, those with WCHT often suffered from metabolic syndrome, as the Ohasama study reported [2]. Metabolic syndrome is also believed to have strong causality in CVD and mortality, and it is considered to cause endothelial dysfunction, resulting in albuminuria. In the third mechanism, those with WCHT had a higher probability of developing SHT than those with NT [12]. SHT is one of the most important risk factors for CVD, CKD, and albuminuria. Finally, it is possible that WCHT itself causes albuminuria, although there is no evidence supporting this direct causality. Moreover, it remains unknown whether albuminuria in WCHT might result in the progression of $\mathrm{CKD}$, whereas albuminuria is considered causal in CKD because albuminuria has been associated with higher incidence of CVD and progression of CKD [29, 30]. The meaning of WCHT, including its association with albuminuria, will be revealed in future studies. 


\section{Compliance with ethical standards}

Conflict of interest The authors declare that they have no conflict of interest.

\section{References}

1. Solak Y, Kario K, Covic A, Bertelsen N, Afsar B, Ozkok A, Wiecek A, Kanbay M. Clinical value of ambulatory blood pressure: Is it time to recommend for all patients with hypertension? Clin Exp Nephrol. 2016;20:14-22.

2. Ohkubo T, Kikuya M, Metoki H, Asayama K, Obara T, Hashimoto J, Totsune K, Hoshi H, Satoh H, Imai Y. Prognosis of "masked" hypertension and "white-coat" hypertension detected by 24-h ambulatory blood pressure monitoring 10-year follow-up from the Ohasama study. J Am Coll Cardiol. 2005;46:508-15.

3. Fagard RH, Cornelissen VA. Incidence of cardiovascular events in white-coat, masked and sustained hypertension versus true normotension: a meta-analysis. J Hypertens. 2007;25:2193-8.

4. Huang Y, Huang W, Mai W, Cai X, An D, Liu Z, Huang H, Zeng J, $\mathrm{Hu} \mathrm{Y,} \mathrm{Xu} \mathrm{D.} \mathrm{White-coat} \mathrm{hypertension} \mathrm{is} \mathrm{a} \mathrm{risk} \mathrm{factor} \mathrm{for} \mathrm{cardio-}$ vascular diseases and total mortality. J Hypertens. 2017;35:677-88.

5. Briasoulis A, Androulakis E, Palla M, Papageorgiou N, Tousoulis D. White-coat hypertension and cardiovascular events: a metaanalysis. J Hypertens. 2016;34:593-9.

6. Kushiro T, Kario K, Saito I, Teramukai S, Sato Y, Okuda Y, Shimada K. Increased cardiovascular risk of treated white coat and masked hypertension in patients with diabetes and chronic kidney disease: the HONEST Study. Hypertens Res. 2017;40:87-95.

7. Gorostidi M, Vinyoles E, Banegas JR, de la Sierra A. Prevalence of white-coat and masked hypertension in national and international registries. Hypertens Res. 2015;38:1-7.

8. Cuspidi C, Rescaldani M, Tadic M, Sala C, Grassi G, Mancia G. White-coat hypertension, as defined by ambulatory blood pressure monitoring, and subclinical cardiac organ damage: a metaanalysis. J Hypertens. 2015;33:24-32.

9. Fukuhara M, Arima H, Ninomiya T, Hata J, Hirakawa Y, Doi Y, Yonemoto K, Mukai N, Nagata M, Ikeda F, Matsumura K, Kitazono T, Kiyohara Y. White-coat and masked hypertension are associated with carotid atherosclerosis in a general population: the Hisayama study. Stroke. 2013;44:1512-7.

10. Cuspidi C, Sala C, Tadic M, Rescaldani M, Grassi G, Mancia G. Is white-coat hypertension a risk factor for carotid atherosclerosis? A review and meta-analysis. Blood Press Monit. 2015;20:57-63.

11. Triantafyllou A, Doumas M, Anyfanti P, Gkaliagkousi E, Zabulis X, Petidis K, Gavriilaki E, Karamaounas P, Gkolias V, Pyrpasopoulou A, Haidich AB, Zamboulis C, Douma S. Divergent retinal vascular abnormalities in normotensive persons and patients with never-treated, masked, white coat hypertension. Am J Hypertens. 2013;26:318-25.

12. Mancia G, Bombelli M, Facchetti R, Madotto F, Quarti-Trevano F, Polo Friz H, Grassi G, Sega R. Long-term risk of sustained hypertension in white-coat or masked hypertension. Hypertension. 2009;54:226-32.

13. Hata J, Fukuhara M, Sakata S, Arima H, Hirakawa Y, Yonemoto K, Mukai N, Kitazono T, Kiyohara Y, Ninomiya T. White-coat and masked hypertension are associated with albuminuria in a general population: the Hisayama Study. Hypertens Res. 2017;40:937-43.

14. Hirayama A, Konta T, Hozawa A, Kawasaki R, Watanabe T, Shibata Y, Kayama T, Fukao A, Kubota I. Slight increase in urinary albumin excretion within the normal range predicts incident hypertension in a community-based Japanese population: the Takahata study. Hypertens Res. 2015;38:56-60.

15. Nagasawa Y, Hasuike Y, Nanami M, Kuragano T, Nakanishi T. Albuminuria and hypertension: the chicken or the egg?. Hypertens Res. 2015;38:8-10.

16. Pierdomenico SD, Cipollone F, Lapenna D, Bucci A, Cuccurullo F, Mezzetti A. Endothelial function in sustained and white coat hypertension. Am J Hypertens. 2002;15:946-52.

17. Gomez-Cerezo J, Rios Blanco JJ, Suarez Garcia I, Moreno Anaya P, Garcia Raya P, Vazquez-Munoz E, Barbado Hernandez FJ. Noninvasive study of endothelial function in white coat hypertension. Hypertension. 2002;40:304-9.

18. Oliveras A, Armario P, Hernandez-Del Rey R, Arroyo JA, Poch E, Larrousse M, Roca-Cusachs A, de la Sierra A. Urinary albumin excretion is associated with true resistant hypertension. J Hum Hypertens. 2010;24:27-33.

19. Martin CA, McGrath BP. White-coat hypertension. Clin Exp Pharmacol Physiol. 2014;41:22-9.

20. Cuspidi C, Sala C, Grassi G, Mancia G. White Coat Hypertension: to Treat or Not to Treat?. Curr Hypertens Rep. 2016;18:80.

21. Grassi G, Seravalle G, Trevano FQ, Dell'oro R, Bolla G, Cuspidi C, Arenare F, Mancia G. Neurogenic abnormalities in masked hypertension. Hypertension. 2007;50:537-42.

22. Fagard RH, Stolarz K, Kuznetsova T, Seidlerova J, Tikhonoff V, Grodzicki T, Nikitin Y, Filipovsky J, Peleska J, Casiglia E, Thijs L, Staessen JA, Kawecka-Jaszcz K. Sympathetic activity, assessed by power spectral analysis of heart rate variability, in white-coat, masked and sustained hypertension versus true normotension. J Hypertens. 2007;25:2280-5.

23. Madsen LB, Rasmussen JK, Moller DS, Nyvad O, Pedersen EB. Heart rate variability in white-coat hypertension. Blood Press Monit. 2008;13:65-71.

24. Martin CA, Cameron JD, Chen SS, McGrath BP. Two hour glucose post loading: a biomarker of cardiovascular risk in isolated clinic hypertension. J Hypertens. 2011;29:749-57.

25. Lymperopoulos A, Rengo G, Koch WJ. Adrenergic nervous system in heart failure: pathophysiology and therapy. Circ Res. 2013;113:739-53.

26. Gillman MW, Kannel WB, Belanger A, D'Agostino RB. Influence of heart rate on mortality among persons with hypertension: the Framingham Study. Am Heart J. 1993;125:1148-54.

27. Penne EL, Neumann J, Klein IH, Oey PL, Bots ML, Blankestijn PJ. Sympathetic hyperactivity and clinical outcome in chronic kidney disease patients during standard treatment. J Nephrol. 2009;22:208-15.

28. Zoccali C, Mallamaci F, Parlongo S, Cutrupi S, Benedetto FA, Tripepi G, Bonanno G, Rapisarda F, Fatuzzo P, Seminara G, Cataliotti A, Stancanelli B, Malatino LS. Plasma norepinephrine predicts survival and incident cardiovascular events in patients with end-stage renal disease. Circulation. 2002;105:1354-9.

29. Chronic Kidney Disease Prognosis C, Matsushita K, van der Velde M, Astor BC, Woodward M, Levey AS, de Jong PE, Coresh J, Gansevoort RT. Association of estimated glomerular filtration rate and albuminuria with all-cause and cardiovascular mortality in general population cohorts: a collaborative metaanalysis. Lancet. 2010;375:2073-81.

30. Levey AS, de Jong PE, Coresh J, El Nahas M, Astor BC, Matsushita K, Gansevoort RT, Kasiske BL, Eckardt KU. The definition, classification, and prognosis of chronic kidney disease: a KDIGO Controversies Conference report. Kidney Int. 2011;80:17-28. 